\title{
Effectiveness of Temporal Gait Event Detection Methods in Detecting Ice- Skating Temporal Events
}

\author{
Aminreza Khandan ${ }^{1 *}$, Jason Carey ${ }^{1}$, and Hossein Rouhani ${ }^{1}$ \\ ${ }^{1}$ Mechanical Engineering, University of Alberta, Edmonton, Canada \\ *khandan@ualberta.ca
}

\begin{abstract}
Objective assessment of an ice skater's motions during training sessions and matches helps coaches to monitor the player performance continuously. The initial step to developing a technology to assess the performance is to detect temporal skating events precisely. Our objective is to investigate the effectiveness of gait event detection methods in detecting skating events using Inertial Measurement Units (IMU). Four highly-cited gait event detection methods using inertial sensors in literature were adopted to detect Skate Strike (SS) and Blades-Off (BO) in skating. Aminian et al. (2002) suggested an algorithmic method based on wavelet analysis to detect heel strikes and toe-offs during gait from the angular velocity of lower limbs. Salarian et al. (2004) and Mariani et al. (2010) respectively, proposed that two negative peaks of the angular velocity of the lower limb and foot are associated with heel strike and toe-off. Mariani et al. (2013) evaluated 24 feature extraction methods to obtain the gait events and suggested that the maximum and minimum of the absolute value of the foot acceleration signal ( II A I| ) can detect heel strike and toe-off in gait better than other signal features. These studies did not elaborate on the definition of the sensors frame or a functional calibration procedure to align these frames with anatomical frames of the body segments, and thus we had to add a presumption. To implement these methods in our studies, we presumed that the angular velocity described in these studies was the angular velocity about the anatomical frame of the segment. Therefore, we virtually rotated the IMU frames and aligned them with the anatomical studies above using a functional calibration procedure suggested in Nazarahari et al. (2019). We attached two IMU (Xsens Technologies, $\mathrm{NL}$ ) on both skates and two on the lower legs of three participants. We also placed two pressure insoles (Pedar system) into their skates, used as a gold standard. The mean and standard deviation of the errors indicate that the most accurate methods are Mariani et al. 2013 (SS: $-0.07 \pm 0.26 \mathrm{sec} \&$ BO: $-0.02 \pm 0.07 \mathrm{sec}$ ) and Mariani et al. 2010 (SS: $-0.02 \pm 0.20 \mathrm{sec} \&$ BO: $0.03 \pm 0.10 \mathrm{sec}$ ). Thus, these methods are effective in finding skating events precisely. However, they are developed for gait analysis and can be further tuned for skating event detection. Fusing two algorithms can also increase assessment precision and accuracy in future studies.
\end{abstract}

Word count: 400 